\title{
Genetic response of Paspalum plicatulum to genome duplication
}

\author{
Emilse Weihmüller • Celina Beltrán • \\ María Sartor · Francisco Espinoza • \\ Claudia Spampinato $\cdot$ Silvina Pessino
}

Received: 16 January 2014/ Accepted: 20 May 2014/Published online: 25 May 2014

(c) Springer International Publishing Switzerland 2014

\begin{abstract}
Paspalum plicatulum is a perennial rhizomatous grass with natural diploid and polyploid cytotypes. In this study, we investigated the occurrence of sequence polymorphisms arising immediately after genome autoduplication in this species. Two mixoploid plants (4C and 7D) were previously obtained through colchicine treatment of seeds generated by open pollination of a diploid plant (H14-2x). Diploid and tetraploid sectors from both mixoploids were dissected to generate two ploidy series $(4 \mathrm{C}$ 2x/4C-4x and 7D-2x/7D-4x). Molecular fingerprints were generated from the maternal plant H14-2x, both ploidy series (4C-2x/4C-4x and 7D-2x/7D-4x), and a tetraploid plant $(\mathrm{C} 1)$ produced by selfing $7 \mathrm{D}-4 \mathrm{x}$. Our results indicate that immediately after polyploidization $P$. plicatulum suffers genetic rearrangements affecting $\sim 28-38 \%$ of the genome. Band gain and loss were equally prevalent at a statistically significant level. At least $5.62 \%$ of the genome experimented recurrent genetic variation in a non-random basis with a confidence of $94.88 \%$. A significant
\end{abstract}

E. Weihmüller · C. Beltrán · S. Pessino $(\bowtie)$

Facultad de Ciencias Agrarias, Universidad Nacional de Rosario, Parque Villarino S/N, S2125ZAA, Zavalla,

Provincia de Santa Fe, Argentina

e-mail: pessino@arnet.com.ar

M. Sartor · F. Espinoza

Facultad de Ciencias Agrarias, Instituto de Botánica del

Nordeste (IBONE, CONICET), Universidad Nacional del

Nordeste, Sargento Cabral 2131, 3400 Corrientes,

Provincia de Corrientes, Argentina

\section{Spampinato}

Facultad de Ciencias Bioquímicas y Farmacéuticas, Centro de

Estudios Fotosintéticos y Bioquímicos (CEFOBI, CONICET),

Universidad Nacional de Rosario, Suipacha 531,

2000 Rosario, Provincia de Santa Fe, Argentina proportion of novel bands (36 out of 195; $18.4 \%$ ) was detected in the $\mathrm{C} 1$ tetraploid plant. Half of these bands were not amplified in either H14-2x or 7D-4x, while the remainders were present in H14-2x but absent in 7D-4x. Our results indicate the occurrence of a considerable number of genetic changes in $P$. plicatulum immediately after polyploidization, some of which were recurrently detected in different independent events. Moreover, we confirmed that after polyploidization, lost ancestral alleles were spontaneously recovered in further generations, a phenomenon previously reported by other research groups.

Keywords Autopolyploidy - Genetic response - Genomic shock $\cdot$ Molecular markers $\cdot$ Paspalum

\section{Introduction}

Approximately 80 years ago, Blakeslee and Avery (1937) induced polyploidy in plants using colchicine, a chemical inhibitor of mitotic cell division. This technique has been successfully used to double the genome in meristematic cells of diploids and interspecific hybrids, producing autoor allopolyploids (Chen and $\mathrm{Ni}$ 2006). Genome-wide genetic analysis of such newly synthesized polyploids using molecular markers is an excellent method for examining short-term plant genomic response to whole genome duplication (Osborn et al. 2003).

Variable results, depending on species, have been obtained from studies of short-term genetic response to allopolyploidization. Using restriction fragment length polymorphism (RFLP) analysis of Brassica synthetic allotetraploids, Song et al. (1995) pioneered the study of ploidy-induced genomic re-organization. Extensive genomic changes, most involving loss and/or gain of parental 
restriction fragments and the appearance of novel fragments, were detected. These changes occurred in self-fertilized progenies from $F_{2}$ through $F_{5}$ generations (Song et al. 1995). Although wheat allotetraploids exhibited $10-15 \%$ genomic alteration in the first hybrid generation, few changes in homoeologous genomes were still occurring by the third generation (Feldman et al. 1997; Shaked et al. 2001). A negligible rate of genomic variation has been reported in allopolyploid cotton and Spartina (Liu et al. 2001; Salmon et al. 2005), while a relatively low polymorphism frequency $(\sim 1 \%)$ has been detected in Arabidopsis allotetraploids (Comai et al. 2000; Madlung et al. 2002).

Although not involving the conflictive collision of two alien genomes, autopolyploidization also appears to induce rapid sequence variation, at least in grasses. Genomic rearrangements were detected in Paspalum sp. when ploidy levels were incrementally increased via hybridization involving unreduced gametes or colchicine treatment (Martelotto et al. 2007). Genetic modifications affected 15-23 and $9.55 \%$ of genomic loci in Paspalum rufum and Paspalum notatum, respectively (Martelotto et al. 2007). Furthermore, in an Eragrostis curvula "back and forth" ploidy series with an isogenic genetic background, Mecchia et al. (2007) reported that nearly $30 \%$ of total loci were polymorphic. These researchers used a natural tetraploid-dihaploid-colchiploid plant series, a unique system that allowed detection of a significant proportion of bands disappearing during dihaploidization and re-appearing upon restoration of polyploidy, or vice versa (Mecchia et al. 2007). Their results provided evidence that some of the genetic modifications were reversible, were highly specific rather than stochastic, and conferred a particular genetic constitution, which was characteristic of the ploidy level (meaning that some of the genomic sequences were modified when the ploidy level descended from $4 \mathrm{x}$ to $2 \mathrm{x}$, but the original genetic structure was recovered when the ploidy level uprised again from $2 \mathrm{x}$ to $4 \mathrm{x}$ ) (Mecchia et al. 2007). In contrast, genetic variation was not detected in dicot species. Ozkan et al. (2006) observed a perfectly additive behaviour in Arabidopsis thaliana autopolyploids. Moreover, in Solanum wild species, Aversano et al. (2013) have not detected microsatellite polymorphisms between synthetic tetraploids and diploid progenitors.

In this study we compared the genetic structure of two diploid/tetraploid plant series of Paspalum plicatulum (brownseed paspalum), a native American rhizomatous perennial grass. Two different mixoploids (experimental codes $4 \mathrm{C}$ and 7D) were independently produced by treating seeds of a diploid maternal plant (H14-2x) with colchicine. Diploid and tetraploid sectors of these mixoploids were selected to generate two different $2 \mathrm{x} / 4 \mathrm{x}$ series. The genomic structures of the two series were then analyzed using amplified fragment length polymorphism (AFLP) and random amplified polymorphic DNA (RAPD) markers and compared with the structure of the maternal plant. A tetraploid derived from selfing of 7D-4x was also examined. Our working hypothesis was that sequence variation in $P$. plicatulum occurs immediately after autopolyploidization, and that changes are specific rather than stochastic.

\section{Materials and methods}

Plant material

The $P$. plicatulum cytotypes used in this project were part of a series, available in our laboratory, which included different ploidies and related genetic backgrounds. The employed set of cytotypes comprised: (a) a natural sexual self-incompatible outcrossing diploid plant (experimental code H14-2x; $2 n=2 x=20$ ); (b) two diploid plants (experimental codes 4C-2x and 7D-2x; $2 n=2 x=20$ ) obtained by dissection of two different mixoploids (4C and 7D) that had been generated by colchicine treatment of H14-2x seeds produced by open pollination; (c) two tetraploid plants (experimental codes $4 \mathrm{C}-4 \mathrm{x}$ and $7 \mathrm{D}-4 \mathrm{x}$; $2 n=4 x=40$ ) derived from the same mixoploids (4C and 7D); and (d) a tetraploid plant obtained after self-pollination of 7D-4x (experimental code $\mathrm{C} 1 ; 2 n=4 x=40$ ). Procedures used to generate the mixoploids and both ploidy series are described in Sartor et al. (2009).

\section{Genomic DNA extraction}

DNA samples were extracted from 3-5 g of young leaves following the protocol of Dellaporta et al. (1983) with modifications recommended by Ortiz et al. (1997). DNA concentrations were measured spectrophotometrically at $260 \mathrm{~nm}$ using a QUBIT fluorometer (Invitrogen, Carlsbad, CA, USA). DNA quality was estimated by measuring the Abs260/280 index.

\section{AFLP markers}

AFLP markers were generated as described by Vos et al. (1995), with the modifications indicated in Martelotto et al. (2007), in order to allow detection of bands by silver staining. Oligonucleotides were designed following the KeyGene protocol (AFLP Protocol for Public Release, Version 2.0, Netherlands, 1994) and synthesized by IDT (Integrated DNA Technologies, Iowa, USA). Preamplification reactions were 
Table 1 AFLP and RAPD primers

\begin{tabular}{|c|c|}
\hline Primer & Sequence \\
\hline \multicolumn{2}{|l|}{ EcoRI/MseI $(+1)$} \\
\hline EcoRI $(+1)$ & 5'-GACTGCGTACCAATTCA-3' \\
\hline MseI $(+1)$ & 5'-GATGAGTCCTGAGTAAA-3' \\
\hline \multicolumn{2}{|l|}{ EcoRI/MseI $(+3)$} \\
\hline EcoRI $(+3)-\mathrm{E} 35$ & 5'-GACTGCGTACCAATTCACA- $3^{\prime}$ \\
\hline EcoRI (+3)-E36 & 5'-GACTGCGTACCAATTCACC-3' \\
\hline EcoRI $(+3)-\mathrm{E} 37$ & 5'-GACTGCGTACCAATTCACG-3' \\
\hline EcoRI $(+3)-\mathrm{E} 40$ & 5'-GACTGCGTACCAATTCAGC-3' \\
\hline EcoRI $(+3)-\mathrm{E} 42$ & 5'-GACTGCGTACCAATTCAGT-3' \\
\hline MseI (+3)-M32 & $5^{\prime}$-GATGAGTCCTGAGTAAAAC-3' \\
\hline MseI (+3)-M33 & 5'-GATGAGTCCTGAGTAAAAG-3 \\
\hline MseI (+3)-M36 & 5'-GATGAGTCCTGAGTAAACC-3' \\
\hline MseI (+3)-M37 & $5^{\prime}$-GATGAGTCCTGAGTAAACG-3' \\
\hline MseI (+3)-M38 & $5^{\prime}$-GATGAGTCCTGAGTAAACT-3' \\
\hline MseI (+3)-M40 & 5'-GATGAGTCCTGAGTAAAGC-3' \\
\hline \multicolumn{2}{|l|}{ RAPD } \\
\hline UBC372 & $5^{\prime}-\mathrm{CCCACTGAC}-3^{\prime}$ \\
\hline UBC373 & $5^{\prime}$-CTGAGGAGT-3' \\
\hline UBC374 & $5^{\prime}$-GGTCAACCC-3' \\
\hline UBC375 & $5^{\prime}-\mathrm{CCGGACACG-} 3^{\prime}$ \\
\hline UBC376 & $5^{\prime}$-CAGGACATC-3' \\
\hline UBC379 & $5^{\prime}$-GGGCTAGGG-3' \\
\hline UBC380 & $5^{\prime}$-AGGAGTGAG-3' \\
\hline UBC383 & $5^{\prime}$-GAGGCGCTG-3' \\
\hline UBC388 & 5'-CGGTCGCGT-3' \\
\hline UBC391 & $5^{\prime}$-GCGAACCTC-3' \\
\hline UBC392 & $5^{\prime}$-CCTGGTGGT-3' \\
\hline UBC399 & $5^{\prime}$-TTGCTGGGC-3' \\
\hline
\end{tabular}

conducted with the primers EcoRI/MseI (+1), which included single selective nucleotides at their $3^{\prime}$ ends (Table 1). For selective amplification, alternative EcoRI/MseI (+3) primers carrying three selective nucleotides at their $3^{\prime}$ ends were used (Table 1). The following 16 primer combinations were tested: M32-E35, M32-E37, M32-E42, M33-E36, M33-E42, M36-E37, M36-E40, M37-E36, M37-E42, M38-E35, M38E36, M38-E42, M40-E36, M40-E37, M40-E40, and M40E42. Several (12) of the primer combinations used did not amplify at all, or showed no amplification in one or more lanes. Finally, 4 primer combinations (M32-E35, M37-E36, M38-E35 and M40-E36), which consistently amplified all samples, were selected for analysis. Amplification products were loaded onto denaturing $5 \%(\mathrm{w} / \mathrm{v})$ acrylamide:bisacrylamide 19:1/7.5 $\mathrm{M}$ urea gels and electrophoresed in TBE buffer (50 mM Tris- $\mathrm{HCl}, 50 \mathrm{mM}$ boric acid, and $1 \mathrm{mM}$ EDTA pH 8). Gels were silver-stained using the Promega DNA Silver Staining System protocol (Promega, Wisconsin, USA).

\section{RAPD markers}

RAPD experiments were carried out following the original technique of Williams et al. (1990), with modifications. Amplifications were carried out on interrun duplicate samples using 12 primers from the British Columbia University RAPD Primer Synthesis Project (Table 1) following the protocol described in CIMMYT Applied Molecular Genetics Laboratory Protocols (www.cimmyt.org). Reaction mixtures were electrophoresed on denaturing $5 \%$ (w/v) acrylamide:bisacrylamide 19:1/7.5 M urea gels. Since these gels can differentiate fragments whose sizes differ in only one nucleotide, the possibility of detecting non-homologous co-migrating bands was drastically reduced. Gels were silver-stained using the same protocol described for AFLP markers. All RAPD profiles were generated twice, and only bands consistently present in both replicates were counted.

Data analysis

To construct a binary data matrix, band presence and absence were designated as 1 and 0 , respectively. Bands with identical mobility were considered to represent the same locus. Similarity coefficients (Jaccard 1908) were calculated from the resulting matrix and subjected to cluster analysis based on UPGMA (unweighted pair group method with arithmetic means) using NTSyS software (Rohlf 2002). Goodness-of-fit Chi squared tests were carried out using the GraphPad software (http://graphpad.com/ quickscalcs/chisquared1). Confidence intervals around observed proportions were calculated following the method described by Newcombe (1998), derived from a procedure outlined by Wilson (1927) with a correction for continuity (http://vassarstats.net/prop1.html).

\section{Results}

Genetic variation and cluster analyses

Molecular markers were used to analyze the genetic structure of the following plants: (1) H14-2x (a natural diploid); (2) 4C-2x and 4C-4x (a diploid/tetraploid series with related genetic backgrounds, derived from H14-2x seeds after colchicine treatment); (3) 7D-2x and 7D-4x (a diploid/tetraploid series with related genetic backgrounds, derived from H14-2x seeds after colchicine treatment); and (4) C1 (a tetraploid obtained after selfing 7D-4x). Sixteen AFLP anchored primer combinations were used to produce amplification profiles; 4 of these (E36-M40, E35-M32, E35-M38, and E36-M37) generated clear repetitive profiles and were used to produce 170 markers randomly 

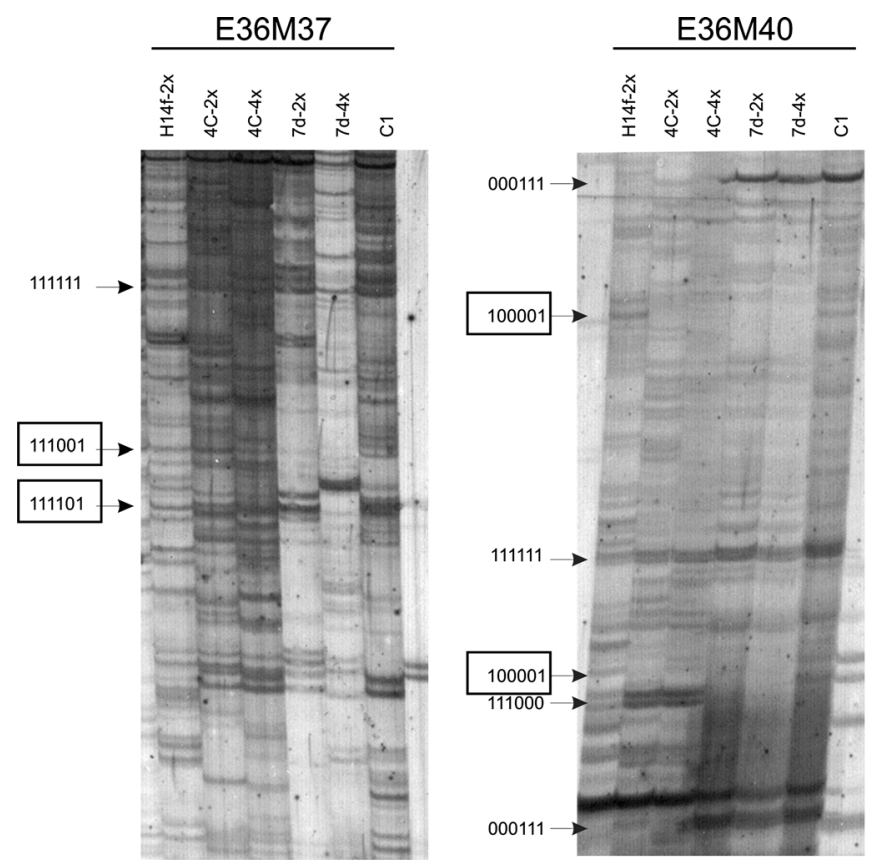

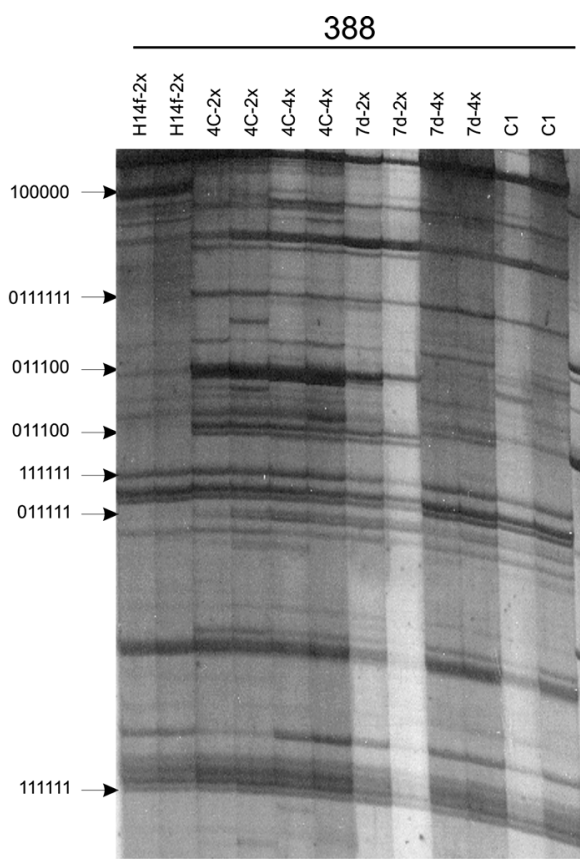

Fig. 1 Banding patterns generated with AFLP and RAPD markers. Left and central panels: amplicons generated by AFLP primer combinations E36M37 and E36M40, respectively. Right panel:

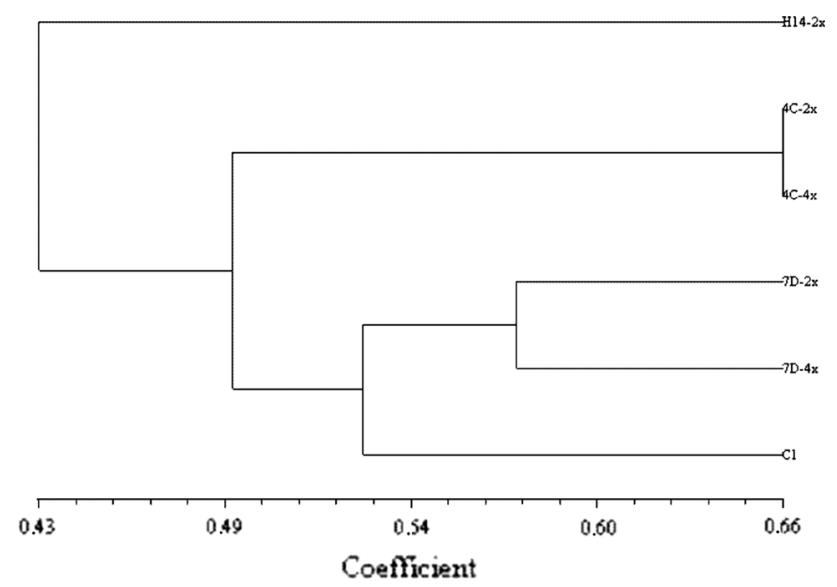

Fig. 2 Genetic similarity dendrogram constructed from the molecular marker data. Genotypes 4C-2x, 4C-4x, 7D-2x, and 7D-4x were generated from H14-2x by open pollination involving different male donors. The occurrence of 0.66 and 0.53 similarity in subsystems $4 \mathrm{C}$ $2 \mathrm{x} / 4 \mathrm{C}-4 \mathrm{x}$ and $7 \mathrm{D}-2 \mathrm{x} / 7 \mathrm{D}-4 \mathrm{x}$ indicate genetic variation occurring during polyploidization. The $\mathrm{C} 1$ polyploid was derived from selfing of 7D-4x. A genetic distance of approximately 0.53 was detected between $\mathrm{C} 1$ and its progenitor

distributed across the $P$. plicatulum genome. We also generated amplification profiles using 12 RAPD primers, 1 of these yielding 25 additional markers (Fig. 1).

The reliability of AFLP profiles was estimated from previously reported data involving $P$. notatum autopolyploids(Martelotto et al. 2007). The error rate inherent to the amplicon generated by decamer UBC388. Arrows indicate examples of detected amplification patterns. Revertant patterns were marked with boxes

genotyping was calculated as recommended by Bonin et al. (2004), as the number of differences per profile divided by the total number of fragments scored per profile (Proportion: $0 ; 95 \%$ CI including continuity correction: $0<p<0.0205$; error rate $<2.05 \%$. We assumed that $P$. plicatulum data analyses maintained equivalent error rates, as the experiments were carried out in the same laboratory with an identical methodology. All RAPD profiles were generated twice, and only consistent bands were kept. Therefore, the error rate of RAPD analysis was considered to be $\sim 0.0 \%$.

Data were transferred into a binary matrix and analyzed with the NTSyS software to produce a genetic similarity dendrogram (Fig. 2). Maternal plant H14-2x was positioned as an outgroup relative to both derived ploidy series (4C-2x/4C-4x and 7D-2x/7D-4x), at a genetic distance of 0.43 . The pair $4 \mathrm{C}-2 \mathrm{x} / 4 \mathrm{C}-4 \mathrm{x}$, produced from mixoploid $4 \mathrm{C}$, clustered together at a distance of 0.66 . The pair 7D-2x/7D$4 \mathrm{x}$, produced from mixoploid 7D, grouped at a distance of 0.57 . Finally, the genotype $\mathrm{C} 1$, which had been produced by selfing of 7D-4x, clustered with the pair 7D-2x/7D-4x at a distance of 0.53 .

Our results revealed a correlation between ploidy increase and the occurrence of sequence variation in $P$. plicatulum. While these variations could be ascribed to either genomic shock produced by sudden increase in nuclear DNA content, or the occurrence of random mutations derived from the use of colchicine, or both, further 
analysis suggested that genomic shock was responsible for at least some of the variation (see below).

Conserved polymorphic patterns in plants with different ploidies and related genetic backgrounds

To determine whether the genetic variation observed between plants with different ploidies and related genetic backgrounds (4C-2x/4C-4x and 7D-2x/7D-4x) involved random loci or was common to both systems, we classified and analyzed all detected polymorphic pattern categories (Table 2).

The number of polymorphic bands arising or disappearing during the transition from $2 \mathrm{x}$ to $4 \mathrm{x}$ was calculated for each ploidy series. Out of 149 molecular markers analyzed in the subsystem 4C-2x/4C-4x, 43 were polymorphic (Proportion: 0.2886; $95 \%$ CI $0.2189<p<0.3695$ ). Of these 43 polymorphic bands, 19 involved band gain (Proportion: 0.4419; $95 \%$ CI $0.2941<p<0.6$ ) and 24 were associated with band loss (Proportion: 0.5581 ; $95 \%$ CI $0.4<p<0.7059)$. The difference in the number of lost and gained bands was not statistically significant $\left(\chi^{2}=0.581 ; d f=1\right.$; two-tailed $p$ value $=0.4458)$. Out of 132 bands recorded in the pair 7D-2x/7D-4x, 51 were polymorphic (Proportion: 0.3864; $95 \%$ CI $0.3041<p<0.4754$ ), with 23 showing band gain (Proportion: 0.451 ; $95 \%$ CI $0.3138<p<0.5955$ ) and 28 exhibiting band loss (Proportion: 0.549; $95 \% \mathrm{CI}$ $0.4045<p<0.6862$ ). Here again, the difference in the number of lost and gained bands was not statistically significant $\quad\left(\chi^{2}=0.490 ; \quad d f=1 ; \quad\right.$ two-tailed $p$ value $=0.4838)$. Our results indicate the occurrence of extensive genetic changes, affecting 28.8-38.6\% of total loci during autopolyploidization, in both systems. Both band loss and band gain were equally prevalent.

The occurrence of conserved polymorphisms, i.e., the same genetic changes in both ploidy systems, was also examined. Thirteen (13) out of 178 markers experimented genetic variation in both systems in concert (Proportion: 0.073; $95 \%$ CI $0.041<p<0.1243$ ) (patterns P16, P21, P30, P36, P39, P43, P45, P46 and P48 in Table 2). From them, ten markers (patterns P16, P21, P36, P39, P43, P45 and P48 in Table 2) underwent the same type of transition, either band loss or gain (Proportion: 0.7692; $95 \%$ CI $0.4598<p<0.9384)$. These ten markers showing a conserved transition represented a genome proportion of 0.0562 (95\% CI $0.0288<p<0.1038$ ). The remaining three markers underwent an opposed type of transition (patterns P30 and P46 in Table 2). Assuming that genetic variation occurred concurrently in both systems at random, the probability of detecting conserved and non-conserved changes would be equal ( $p=0.50$; expected value for conserved changes: 6.5; expected value for non-conserved changes: 6.5). A goodness-of-fit Chi squared test to check the possibility of non-random genetic variation rendered a value $\chi^{2}: 3.769 ; d f=1$; two-tailed $\mathrm{p}$ value: 0.0522 . Therefore, our results indicate that changes are non-random with a confidence of $94.88 \%$.

Six of the ten conserved changes involved band loss (Proportion: 0.60; $95 \%$ CI $0.2737<p<0.8631$ ) and four corresponded to band gain (Proportion: 0.40; $95 \%$ CI $0.1369<p<0.7263$ ). The difference between the rate of occurrence of band loss and band gain was not statistically significant $\quad\left(\chi^{2}=0.400 ; \quad d f=1 ; \quad\right.$ two-tailed $p$ value $=0.5271)$. A Chi squared test aimed at analyzing if band gain or band loss occurred in a non-random basis was not applicable, due to the low number of markers detected for each of the individual patterns.

Considering the $4 \mathrm{C}-2 \mathrm{x} / 4 \mathrm{C}-4 \mathrm{x}$ system, 10 of the 43 observed polymorphisms also occurred in the other system and were thus conserved (Proportion: 0.2326; $95 \% \mathrm{CI}$ $0.1229<p<0.39$ ). When the $7 \mathrm{D}-2 \mathrm{x} / 7 \mathrm{D}-4 \mathrm{x}$ system was analyzed, 10 out of 51 observed polymorphisms occurred concurrently in the other ploidy series (Proportion: 0.1961; $95 \%$ CI $0.1029<p<0.3355)$. It should be noted, however, that mixoploids $4 \mathrm{C}$ and $7 \mathrm{D}$ derived from different male donors, as self-incompatible outcrossing H14-2x was subjected to open pollination to guarantee a large seed set. This situation may have masked further changes affecting the same loci.

\section{Revertant banding patterns}

Interesting polymorphic pattern types were observed in the C1 plant, which originated from selfing of 7D-4x. Eighteen bands present in the diploid ancestor H14-2x, representing a proportion of 0.1782 , (95\% CI $0.1118<p<0.2697$ ) disappeared in the pair 7D-2x/7D-4x, but reappeared in $\mathrm{C} 1$. Similar results regarding recovery of ancestral bands have been reported by Song et al. (1995) in synthetic Brassica allopolyploids. Genetic reversion was also detected in $E$. curvula autotetraploids by Mecchia et al. (2007). In addition, 18 novel bands that had never been detected in the ancestor nor the progenitor appeared in the $\mathrm{C} 1$ plant.

\section{Discussion}

Extensive studies on the molecular response of the plant genome to polyploidization were carried out mainly from synthetic allopolyploids. By contrast, less is known about the consequences of autopolyploidization. The available articles were reviewed in Parisod et al. (2010), Tayalé and Parisod (2013). They were focused on analyzing genetic variation in recently synthesized autopolyploids of $P$. notatum (Martelotto et al. 2007), P. rufum (Martelotto et al. 
Table 2 Types and number of detected amplification patterns

\begin{tabular}{|c|c|c|c|c|c|c|c|c|c|}
\hline Pattern code & H14-2x ${ }^{a}$ & $4 c-2 x^{a}$ & $4 c-4 x^{a}$ & $7 d-2 x^{a}$ & $7 d-4 x^{a}$ & $\mathrm{C} 1^{\mathrm{a}}$ & Total $^{\mathrm{b}}$ & $\mathrm{AFLP}^{\mathrm{c}}$ & RAPDs $^{\mathrm{d}}$ \\
\hline P1 & 1 & 1 & 1 & 1 & 1 & 1 & 44 & 31 & 13 \\
\hline P2 & 0 & 1 & 1 & 0 & 0 & 0 & 13 & 12 & 1 \\
\hline P3 & 0 & 1 & 1 & 1 & 1 & 1 & 15 & 12 & 3 \\
\hline P4 & 1 & 1 & 0 & 0 & 0 & 1 & $1^{*}$ & 1 & 0 \\
\hline P5 & 0 & 0 & 1 & 1 & 1 & 1 & 3 & 3 & 0 \\
\hline P6 & 1 & 0 & 0 & 0 & 0 & 1 & $7 *$ & 7 & 0 \\
\hline P7 & 1 & 1 & 0 & 0 & 0 & 0 & 3 & 3 & 0 \\
\hline P8 & 1 & 1 & 1 & 0 & 0 & 1 & $4 *$ & 4 & 0 \\
\hline P9 & 0 & 1 & 1 & 1 & 1 & 0 & 2 & 1 & 1 \\
\hline P10 & 0 & 1 & 0 & 0 & 0 & 1 & $3 \#$ & 3 & 0 \\
\hline P11 & 1 & 0 & 0 & 0 & 0 & 0 & 6 & 5 & 1 \\
\hline $\mathrm{P} 12$ & 0 & 1 & 0 & 0 & 0 & 0 & 6 & 6 & 0 \\
\hline P13 & 1 & 1 & 1 & 0 & 0 & 0 & 5 & 5 & 0 \\
\hline P14 & 1 & 1 & 1 & 1 & 0 & 0 & 1 & 1 & 0 \\
\hline P15 & 0 & 0 & 0 & 1 & 1 & 1 & 1 & 1 & 0 \\
\hline P16 & 0 & 1 & 0 & 1 & 0 & 1 & $3+\#$ & 3 & 0 \\
\hline P17 & 0 & 1 & 1 & 0 & 0 & 1 & 4\# & 4 & 0 \\
\hline P18 & 1 & 0 & 1 & 1 & 1 & 1 & 4 & 2 & 2 \\
\hline P19 & 0 & 1 & 1 & 1 & 0 & 1 & $2 \#$ & 2 & 0 \\
\hline P20 & 0 & 0 & 0 & 1 & 0 & 0 & 8 & 8 & 0 \\
\hline $\mathrm{P} 21$ & 0 & 0 & 1 & 0 & 1 & 0 & $1+$ & 1 & 0 \\
\hline $\mathrm{P} 22$ & 0 & 0 & 0 & 1 & 0 & 1 & $2 \#$ & 2 & 0 \\
\hline $\mathrm{P} 23$ & 0 & 0 & 1 & 0 & 0 & 0 & 3 & 3 & 0 \\
\hline P24 & 1 & 1 & 1 & 0 & 1 & 1 & 2 & 2 & 0 \\
\hline $\mathrm{P} 25$ & 1 & 1 & 1 & 1 & 1 & 0 & 6 & 6 & 0 \\
\hline P26 & 1 & 0 & 0 & 1 & 0 & 1 & $2^{*}$ & 2 & 0 \\
\hline P27 & 1 & 0 & 0 & 1 & 1 & 1 & 2 & 2 & 0 \\
\hline P28 & 0 & 1 & 1 & 0 & 1 & 1 & 1 & 1 & 0 \\
\hline P29 & 1 & 0 & 1 & 0 & 0 & 1 & $2^{*}$ & 2 & 0 \\
\hline P30 & 0 & 0 & 1 & 1 & 0 & 0 & 1 & 1 & 0 \\
\hline P31 & 0 & 0 & 0 & 0 & 1 & 1 & 3 & 3 & 0 \\
\hline P32 & 0 & 0 & 0 & 0 & 1 & 0 & 7 & 7 & 0 \\
\hline P33 & 0 & 0 & 0 & 1 & 1 & 0 & & 1 & 0 \\
\hline P34 & 0 & 0 & 0 & 0 & 0 & 1 & $4 \#$ & 4 & 0 \\
\hline P35 & 1 & 1 & 0 & 1 & 1 & 1 & 2 & 2 & 0 \\
\hline P36 & 0 & 1 & 0 & 1 & 0 & 0 & $1+$ & 1 & 0 \\
\hline P37 & 1 & 0 & 0 & 1 & 0 & 0 & 2 & 2 & 0 \\
\hline P38 & 0 & 1 & 1 & 0 & 1 & 1 & 2 & 2 & 0 \\
\hline P39 & 1 & 1 & 0 & 1 & 0 & 1 & $1+*$ & 1 & 0 \\
\hline P40 & 1 & 1 & 1 & 1 & 0 & 1 & $1^{*}$ & 1 & 0 \\
\hline P41 & 1 & 0 & 1 & 0 & 0 & 0 & 2 & 1 & 1 \\
\hline P42 & 1 & 0 & 0 & 0 & 1 & 1 & 1 & 1 & 0 \\
\hline P43 & 1 & 0 & 1 & 0 & 1 & 1 & $1+$ & 1 & 0 \\
\hline P44 & 0 & 1 & 1 & 1 & 0 & 0 & 3 & 1 & 2 \\
\hline P45 & 0 & 0 & 1 & 0 & 1 & 1 & $2+$ & 2 & 0 \\
\hline P46 & 0 & 1 & 0 & 0 & 1 & 1 & 2 & 2 & 0 \\
\hline P47 & 1 & 1 & 0 & 1 & 1 & 0 & 1 & 1 & 0 \\
\hline P48 & 0 & 1 & 0 & 1 & 0 & $\mathrm{n}$ & $1+$ & 1 & 0 \\
\hline
\end{tabular}


Table 2 continued

\begin{tabular}{|c|c|c|c|c|c|c|c|c|c|}
\hline Pattern code & $\mathrm{H} 14-2 \mathrm{x}^{\mathrm{a}}$ & $4 c-2 x^{a}$ & $4 c-4 x^{a}$ & $7 d-2 x^{a}$ & $7 d-4 x^{a}$ & $\mathrm{C} 1^{\mathrm{a}}$ & Total $^{\mathrm{b}}$ & AFLP $^{c}$ & RAPDs $^{\mathrm{d}}$ \\
\hline P49 & 1 & 1 & 1 & 0 & 1 & 0 & 1 & 0 & 1 \\
\hline
\end{tabular}

${ }^{a}$ Patterns type were represented by binary code. 1: band presence; 0 : band absence; n: not determined

$\mathrm{b}$ Total number of markers showing the corresponding pattern. +: bands showing a recurrent behaviour in both ploidy systems $(4 \mathrm{C} 2 \mathrm{x} / 4 \mathrm{C} 4 \mathrm{x}$ and 7D2x/7D4x); * bands present in H14-2x but absent in 7D-4x and reappearing in C1 (revertant bands); \# bands absent in both H14-2x and 7D$4 \mathrm{x}$ and appearing in $\mathrm{C} 1$ (novel bands)

${ }^{c}$ Number of markers originated from AFLP experiments showing a particular pattern

${ }^{\mathrm{d}}$ Number of markers originated from RAPD experiments showing a particular pattern

2007) and A. thaliana (Ozkan et al. 2006). Similar studies were carried out in E. curvula (Mecchia et al. 2007), and Solanum sp (Aversano et al. 2013). Interestingly, variable levels of genomic response were reported in grasses (Martelotto et al. 2007; Mecchia et al. 2007), but not in dicot species (Ozkan et al. 2006; Aversano et al. 2013).

Here, we used a novel approach to investigate the grass genome response to autopolyploidization by comparing two independent $2 \mathrm{x}-4 \mathrm{x}$ series originated from $P$. plicatulum mixoploids with related genetic backgrounds. The use of such methodology allowed us to conclude that part of the short-term genome variation observed after polyploidization in this species is non-random. Our results might contribute to elucidate the nature of genetic variations occurring after whole genome autoduplication.

Depending on the series used, we observed a genetic variation involving $28-38 \%$ of the analyzed loci. Our results are comparable to those reported for related grasses such as $P$. notatum, $P$. rufum and $E$. curvula, where genetic variation following autopolyploidization was associated with 15-23, 9, and 29-32\%, respectively, of studied loci (Martelotto et al. 2007; Mecchia et al. 2007). In the previous studies, however, the ratio of band loss/band gain was always unbalanced. For example, Martelotto et al. (2007) reported that during $P$. rufum and $P$. notatum $2 \mathrm{x}-4 \mathrm{x}$ transitions, 100 and $88.5 \%$ of the polymorphisms involved band loss, respectively. Conversely, in E. curvula $2 \mathrm{x}-4 \mathrm{x}$ transitions, nearly $70 \%$ of the polymorphisms were associated with band gain (Mecchia et al. 2007). In P. plicatulum, neither band loss nor band gain predominated during $2 \mathrm{x}-4 \mathrm{x}$ transitions at a significant level.

A relevant question is whether the colchicine treatment used to produce $P$. plicatulum mixoploids could have caused independent genetic rearrangements in the diploid and tetraploid constituents of the ploidy series. If so, these colchicine-induced mutations could have erroneously been perceived as changes originated from the $2 \mathrm{x}-4 \mathrm{x}$ transition. Unfortunately, comparison of H14-2x with 4D-2x or 7D-2x to estimate the occurrence of random mutations due to colchicine is uninformative because the mixoploids were generated by open pollination. Consequently, we cannot rule out the possibility that some of the observed variation may have arisen from the treatment. However, the use of two replicates offers first hints on repeated changes. The majority of the polymorphic sites affected in both systems underwent the same pattern of genetic change. A Chi squared test indicated that such variation is non-random, with a confidence of $94.88 \%$. These results imply that at least a significant proportion of polymorphisms detected during the $2 \mathrm{x}-4 \mathrm{x}$ transition were related to a genomic shock triggered by an increase in the number of genomic complements within the nucleus rather than to the effect of colchicine.

Several unexpected markers were observed in the $\mathrm{C} 1$ tetraploid, involving 36 out of 195 analyzed loci. Eighteen (18) of them were novel, while the remaining 18 had been already detected in the ancestor H14-2x. The 18 novel bands that appeared for the first time in $\mathrm{C} 1$ could have been originated from genome confrontation, since $\mathrm{C} 1$, in contrast to the other autopolyploids analyzed here, has gone through meiosis. Regarding revertant bands, all of them were detected from AFLP amplification. Therefore, reappearance could have been originated from epigenetic changes affecting EcoRI, since this enzyme is sensitive to $\mathrm{CpG}$ methylation when the restriction site overlaps the methylation sequence. In an extensive survey of sequence variation after wide hybridization and allopolyploidy in wheat, Shaked et al. (2001) mentioned the occurrence of five novel bands that appeared in F1 and dissapeared again in the allopolyploid, and seven cases of bands that were present in the parents, dissapeared in $\mathrm{F} 1$, and reappeared in the allotetraploid. The authors suggested that these deviations from additivity were probably caused by changes in methylation. Similar revertant patterns were further reported in Ozkan et al. (2001).

However, special attention should be paid to the type and number of markers analyzed. All revertant patterns reported here were originated from AFLP bands, but while the counted RAPD bands were 25, the AFLP ones were 170. The proportion of revertant bands observed from AFLP experiments was 0.1059 (18/170), indicating a $95 \%$ CI of $0.0657<p<0.1646$ (Newcombe 1998). The proportion of revertant bands observed from RAPD experiments was 0 (0/ 
25 ), with a $95 \%$ CI of $0<p<0.1658$. The overlapping of $\mathrm{CI}$ indicates that the number of RAPD markers analyzed might have been too low to discard similar proportions of revertant bands originated from both marker types. Moreover, analogous revertant genomic changes occurring during polyploidization have been previously detected using alternative techniques in newly-synthetized Brassica napus allopolyploids (Song et al. 1995) and E. curvula autopolyploids (Mecchia et al. 2007). Part of the revertant variation detected by Song et al. (1995) was revealed by RFLP analysis using HindIII, a methylation insensitive enzyme. Mecchia et al. (2007) reported revertant bands originated from RAPD analysis, which rules out the possibility of an epigenetic origin. These authors recovered 18 revertant bands included in six co-migrating groups and sequenced them (Mecchia et al. 2007). Intragroup identity was confirmed for 17 of them, since one failed amplification (Mecchia et al. 2007).

Results commented above indicate that the recovery of missing bands might be at least partially addressed to a still unknown mechanism The possibility of the use of an ancestral RNA-sequence cache, as proposed by Lolle et al. (2005), should be considered. Another hypothesis could be based on non-random movement of transposons/retrotransposons. However, these explanations remain highly speculative, since further experimental information is required. Isolation, cloning and sequencing of revertant bands might help to elucidate the nature of these intriguing variation.

Acknowledgments This research was supported by Agencia Nacional de Promoción Científica y Tecnológica (ANPCyT), Argentina (Projects PICT 2007-00476 and PICT 2011-1269), and Consejo Nacional de Investigaciones Científicas y Técnicas (CONICET), Argentina (Project PIP No. 11220090100613). M. Sartor is a CONICET postdoctoral fellow, and F. Espinoza, C. Spampinato, and S. Pessino are CONICET research staff members.

\section{References}

Aversano R, Caruso I, Aronne G, De Micco V, Scognamiglio N, Carputo D (2013) Stochastic changes affect Solanum wild species following autopolyploidization. J Exp Bot 64(2):625635

Blakeslee AF, Avery AG (1937) Methods of inducing doubling of chromosomes in plants by treatment with colchicine. J Hered 28:393-411

Bonin A, Bellemain E, Bronken Eidesen P, Pompanon F, Brochmann C, Taberlet P (2004) How to track and assess genotyping errors in population genetics studies. Mol Ecol 13:3261-3273

Chen ZJ, Ni Z (2006) Mechanisms of genomic rearrangement and gene expression changes in plant polyploids. Bioassays 28:240-252

Comai L, Tyagi AP, Winter K, Holmes-Davis R, Reynolds SH, Stevens Y et al (2000) Phenotypic instability and rapid gene silencing in newly formed Arabidopsis allotetraploids. Plant Cell 12:1551-1568

Dellaporta SL, Wood J, Hicks JB (1983) A plant DNA minipreparation. Version II. Plant Mol Biol Rep 1(1983):19-21
Feldman M, Liu B, Segal G, Abbo S, Levy AA, Vega JM (1997) Rapid elimination of low-copy DNA sequences in polyploid wheat: a possible mechanism for differentiation of homoeologous chromosomes. Genetics 147:1381-1387

Liu B, Brubaker CL, Mergeai G, Cronn RC, Wendel JF (2001) Polyploid formation in cotton is not accompanied by rapid genomic changes. Genome 44:321-330

Lolle SJ, Victor JL, Young JM, Pruitt R (2005) Genome-wide nonMendelian inheritance of extra-genomic information in Arabidopsis. Nature 434:505-509

Madlung A, Masuelli RW, Watson B, Reynolds SH, Davison J, Comai L (2002) Remodeling of DNA methylation and phenotypic and transcriptional changes in synthetic Arabidopsis allotetraploids. Plant Physiol 129:733-746

Martelotto LG, Ortiz JPA, Stein J, Espinoza F, Quarin CL, Pessino SC (2007) Genome rearrangements derived from autopolyploidization in Paspalum sp. Plant Sci 172:970-977

Mecchia MA, Ochogavía A, Selva JP, Laspina N, Felitti S, Martelotto LG et al (2007) Genome polymorphisms and gene differential expression in a 'back-and-forth' ploidy-altered series of weeping lovegrass (Eragrostis curvula). J Plant Physiol 164:1051-1061

Newcombe RG (1998) Two-sided confidence intervals for the single proportion: comparison of seven methods. Stat Med 17:857-872

Ortiz JPA, Pessino SC, Leblanc O, Hayward MD, Quarin CL (1997) Genetic fingerprinting for determining the mode of reproduction in Paspalum notatum, a subtropical apomictic forage grass. Theor Appl Genet 95:850-856

Osborn TC, Pires JC, Birchler JA, Auger DL, Chen ZJ, Lee H-S et al (2003) Understanding mechanisms of novel gene expression in polyploids. Trends Genet 19:141-147

Ozkan H, Levy AA, Feldman M (2001) Allopolyploidy-induced rapid genome evolution in the wheat (Aegilops-Triticum) group. Plant Cell 13:1735-1747

Ozkan H, Tuna M, Galbraith DW (2006) No DNA loss in autotetraploids of Arabidopsis thaliana. Plant Breed 125: 288-291

Parisod C, Holderegger R, Brochmann C (2010) Evolutionary consequences of autopolyploidy. New Phytol 186:5-17

Rohlf FJ (2002) NTSYSpc: numerical taxonomy system, version 2.1. Exeter Publishing, Setauket

Salmon A, Ainouche ML, Wendel JF (2005) Genetic and epigenetic consequences of recent hybridization and polyploidy in Spartina (Poaceae). Mol Ecol 14:1163-1175

Sartor ME, Quarin CL, Espinoza F (2009) Mode of reproduction of colchicine-induced Paspalum plicatulum tetraploids. Crop Sci 49:1270-1276

Shaked H, Kashkush K, Ozkan H, Feldman M, Levy AA (2001) Sequence elimination and cytosine methylation are rapid and reproducible responses of the genome to wide hybridization and allopolyploidy in wheat. Plant Cell 13:1749-1759

Song K, Lu P, Tang K, Osborn TC (1995) Rapid genome change in synthetic polyploids of Brassica and its implications for polyploid evolution. Proc Natl Acad Sci USA 92:7719-7723

Tayalé A, Parisod C (2013) Natural pathways to polyploidy in plants and consequences for genome reorganization. Cytogenet Genome Res 140(2-4):79-96

Vos P, Hogers R, Bleeker M, Reijans M, Van de Lee T, Hornes M et al (1995) AFLP: a new concept for DNA fingerprinting. Nucl Acids Res 23:4407-4414

Williams JGK, Kubelik AR, Livak KJ, Rafalski JA, Tingey SV (1990) DNA polymorphisms amplified by arbitrary primers are useful as genetic markers. Nucl Acids Res 18:6531-6535

Wilson EB (1927) Probable inference, the law of succession, and statistical inference. Journal of the American Statistical Association 22:209-212 